\title{
Description of analyzing power and $(p, n)$ reaction by a global dispersive coupled-channel optical model potential
}

Cite as: AIP Conference Proceedings 1235, 43 (2010); https://

doi.org/10.1063/1.3442630

Published Online: 01 June 2010

W. L. Sun, L. J. Hao, E. Sh Soukhovitskii, R. Capote, and J. M. Quesada

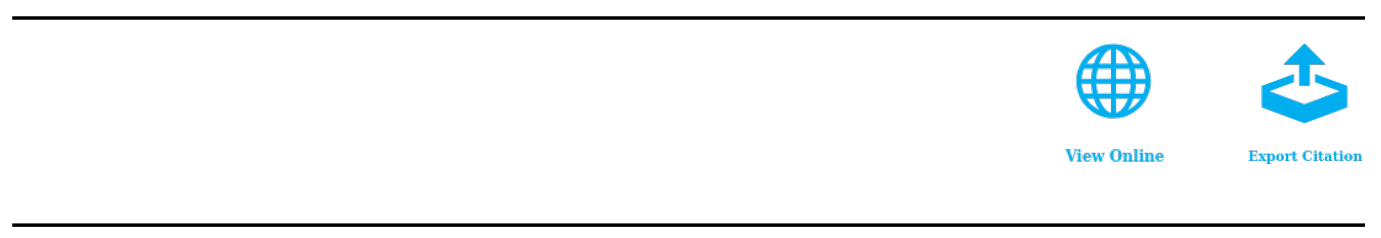

\section{Lock-in Amplifiers up to $600 \mathrm{MHz}$

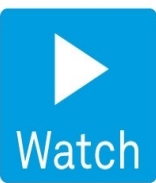

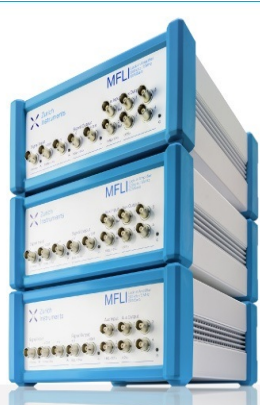




\title{
Description of analyzing power and (p,n) reaction by a global dispersive coupled-channel optical model potential
}

\author{
W. L. Sun*, L. J. Hao ${ }^{\dagger}$, E. Sh Soukhovitskii**, R. Capote ${ }^{*}$ and J. M. \\ Quesada $§$ \\ *Institute of Applied Physics and Computational Mathematics, Beijing 100088, China \\ ${ }^{\dagger}$ Department of Applied Quantum Physics and Nuclear Engineering, Kyushu University, Fukuoka \\ 8190395, Japan \\ ** Joint Institute for Energy and Nuclear Research, Minsk-Sosny 220109, Belarus \\ ${ }^{\ddagger}$ Nuclear Data Section, International Atomic Energy Agency, Vienna A-1400, Austria \\ ${ }^{\S}$ Departamento de Fisica Atomica, Molecular y Nuclear, Universidad de Sevilla, Ap. 1065, \\ E-41080 Sevilla, Spain
}

\begin{abstract}
A global dispersive coupled-channel optical model potential is used to analyze the proton elastic/inelastic analyzing power data and $(p, n)$ data in order to investigate the prediction power of this potential. The numerical calculations show good agreement with available experimental data for proton elastic data and overall agreement for proton inelastic data. The results also showed that present global potential is approximately Lane-consistent, and can predict well the (p,n) cross sections.
\end{abstract}

Keywords: Coupled-Channel, Analyzing power, Isobaric Analog State, Lane consistence PACS: $24.10 . H t, 24.10 . \mathrm{Eq}, 25.40 .-\mathrm{h}, 29.85 .-\mathrm{c}$

\section{INTRODUCTION}

A global dispersive coupled-channel optical model potential for deformed even-even nuclei from $A=24-122$ has been derived in our previous work [1] to describe neutron and proton scattering on deformed nuclei. This global potential was proved to be of good prediction power for elastic and inelastic angular distributions. However further demonstration of its prediction power is needed for applications. One is to test the reproduction quality of of analyzing power data. Many calculations had demonstrated that the global spherical optical model potential, such as Koning and Delaroche[2] potential, is of such a capability, however only for the case of elastic analyzing power.

It is also interesting to check the predictive ability of this potential for the charge exchange (p, n) reaction to the isobaric analog state (IAS) of target ground state. Such an IAS transition is well known as quasielastic scattering, since the total angular momentum and parity transferred are $\Delta J^{\pi}=0^{+}$and the initial and final states are of similar nature except for the replacement of a neutron by a proton. This replacement corresponds an isospin-flip in isospin representation. Based on Lane model[3], the central nucleon-nucleus potential (with Coulomb interaction switched off) can be written as the sum of an isoscalar component and an isovector coupling component, where the latter contributes the proton and neutron elastic scattering as well as the charge exchange 
$(\mathrm{p}, \mathrm{n})$ reaction. However such an isovector part gives negligible contribution to elastic scattering, but dominates the forward $(\mathrm{p}, \mathrm{n})$ angular distributions and spectra. Therefore extending the analyzing for $(\mathrm{p}, \mathrm{n})$ reaction provides tests of isospin as a quantum number as well as isospin-dependence of nucleon-nucleus potential. This work is a supplemental calculation to our previous work, aimed at showing how the the prediction power is for the analyzing power data and the $(\mathrm{p}, \mathrm{n})$ data by using our global potential in order to establish a well-justified optical potential for analyzing the available experimental data.

\section{DISPERSIVE COUPLED-CHANNEL OPTICAL MODEL POTENTIAL}

The details of our global dispersive coupled-channel optical model potential had been described before[1], therefore only the outline is mentioned briefly below.

With account of the deformed nuclear shapes, the coupled-channel optical model potential followed the standard Woods-Saxon form with conventional definition for the symbols and geometrical form factors[4]:

$$
\begin{aligned}
V(r, & \left.R\left(\theta^{\prime}, \varphi^{\prime}\right), E\right)= \\
- & {\left[V_{\mathrm{v}}(E)+\mathrm{iW}_{\mathrm{v}}(\mathrm{E})+\Delta \mathrm{V}_{\mathrm{v}}^{\mathrm{Coul}}(\mathrm{E})\right] \mathrm{f}_{\mathrm{WS}}\left[\mathrm{r}, \mathrm{R}_{\mathrm{v}}\left(\theta^{\prime}, \varphi^{\prime}\right)\right] } \\
- & {\left[V_{\mathrm{s}}(E)+\mathrm{iW}_{\mathrm{s}}(\mathrm{E})+\Delta \mathrm{V}_{\mathrm{s}}^{\mathrm{Coul}}(\mathrm{E})\right] \mathrm{g}_{\mathrm{WS}}\left[\mathrm{r}, \mathrm{R}_{\mathrm{s}}\left(\theta^{\prime}, \varphi^{\prime}\right)\right] } \\
- & \left(\frac{\hbar}{\mu_{\pi c}}\right)^{2}\left[V_{\mathrm{so}}(E)+\mathrm{iW}_{\mathrm{so}}(\mathrm{E})\right] \frac{1}{\mathrm{r}} \frac{\mathrm{d}}{\mathrm{dr}} \mathrm{f}_{\mathrm{WS}}\left[\mathrm{r}, \mathrm{R}_{\mathrm{so}}\left(\theta^{\prime}, \varphi^{\prime}\right)\right] \\
& \times \sigma \cdot \mathbf{L}+V_{\mathrm{Coul}}\left[r, R_{\mathrm{c}}\left(\theta^{\prime}, \varphi^{\prime}\right)\right],
\end{aligned}
$$

The Coulomb potential $V_{\text {Coul }}\left[r, R_{\mathrm{c}}\left(\theta^{\prime}, \varphi^{\prime}\right)\right]$ is calculated using a spherical term plus a higher multipole expansion of charged ellipsoid with a uniform charge density, as suggested by Bassel et al.[5]. The details had been described in Ref.[6].

Based on the dispersion relation theory $[7,8]$, the real potentials are written as[9, 10]:

$$
\begin{gathered}
V_{\mathrm{v}}(E)=V_{\mathrm{HF}}(E)+\Delta V_{\mathrm{v}}(E)=A_{\mathrm{HF}} \mathrm{e}^{-\lambda_{\mathrm{HF}}\left(\mathrm{E}-\mathrm{E}_{\mathrm{f}}\right)}+\Delta \mathrm{V}_{\mathrm{v}}(\mathrm{E}) . \\
V_{\mathrm{s}}(E)=\Delta V_{\mathrm{s}}(E) . \\
V_{\mathrm{so}}(E)=V_{\mathrm{so}} \mathrm{e}^{-\lambda_{\mathrm{so}}\left(\mathrm{E}-\mathrm{E}_{\mathrm{f}}\right)}+\Delta \mathrm{V}_{\mathrm{so}}(\mathrm{E})
\end{gathered}
$$

where $A_{\mathrm{HF}}, \lambda_{\mathrm{HF}}, V_{\mathrm{so}}$ and $\lambda_{\mathrm{so}}$ are undetermined parameters, $E_{\mathrm{f}}$ is the Fermi energy.

The terms $\Delta V_{\mathrm{v}}(E), \Delta V_{\mathrm{s}}(E)$ and $\Delta V_{\mathrm{so}}(E)$, so-called the dispersive correction terms, are calculated using the dispersion relation:

$$
\Delta V(E)=\frac{\mathscr{P}}{\pi} \int_{-\infty}^{+\infty} \frac{W\left(E^{\prime}\right)}{E^{\prime}-E} \mathrm{~d} E^{\prime}
$$

where the symbol $\mathscr{P}$ denotes that the principal value of the integral should be taken. 
The energy dependence for the imaginary terms are represented as [11, 12]:

$$
\begin{gathered}
W_{\mathrm{v}}(E)=A_{\mathrm{v}} \frac{\left(E-E_{\mathrm{f}}\right)^{S}}{\left(E-E_{\mathrm{f}}\right)^{S}+B_{\mathrm{v}}^{S}} \\
W_{\mathrm{s}}(E)=A_{\mathrm{s}} \frac{\left(E-E_{\mathrm{f}}\right)^{S}}{\left(E-E_{\mathrm{f}}\right)^{S}+B_{\mathrm{s}}^{S}} \mathrm{e}^{-C_{\mathrm{s}}\left|E-\mathrm{E}_{\mathrm{f}}\right|}, \\
W_{\mathrm{so}}(E)=A_{\mathrm{so}} \frac{\left(E-E_{\mathrm{f}}\right)^{S}}{\left(E-E_{\mathrm{f}}\right)^{S}+B_{\mathrm{so}}^{S}},
\end{gathered}
$$

where $A_{\mathrm{v}}, B_{\mathrm{v}}, A_{\mathrm{s}}, B_{\mathrm{s}}, C_{\mathrm{s}}, A_{\mathrm{so}}$ and $B_{\mathrm{so}}$ are undetermined parameters.

The isospin dependence of the potential is considered in real volume $V_{\mathrm{HF}}(E)$ and imaginary surface $W_{\mathrm{s}}(E)$ potentials as follows:

$$
A_{\mathrm{HF}}=V_{0}+(-1)^{Z^{\prime}+1} C_{\mathrm{viso}} \frac{N-Z}{A}, A_{\mathrm{s}}=W_{0}+(-1)^{Z^{\prime}+1} C_{\mathrm{wiso}} \frac{N-Z}{A} .
$$

With the above dispersive consideration for each potential term, the potential of Eq. (1) is called as dispersive coupled-channel optical model potential.

The potential parameters for deformed even-even nuclei in the mass range of $\mathrm{A}=24$ 122 for incident energy up to $200 \mathrm{MeV}$ had been searched and given in Table 2 of the previous work[1]. The searching was performed by the OPTMAN code[13].

\section{RESULTS AND DISCUSSIONS}

\section{Analyzing Power}

The previous work had shown the predictions of proton analyzing power for ${ }^{56} \mathrm{Fe}$ and ${ }^{120} \mathrm{Sn}$, here we present some more calculations for other nuclei: ${ }^{24} \mathrm{Mg},{ }^{28} \mathrm{Si},{ }^{60} \mathrm{Ni}$ and ${ }^{90} \mathrm{Zr}$, as shown in Fig. 1, 2, 3, 4 respectively, in order to indicate how the predictive power of this global potential is for analyzing power data. As the OPTMAN code has no option to calculate analyzing power, the ECIS06t code[14], with three levels coupled, was used for such calculations.

Firstly, our global potential gave generally good predictions of the proton elastic $A_{y}$ data for ${ }^{24} \mathrm{Mg}$, except for low incident energy $15.0 \mathrm{MeV}$ and for $49.2 \mathrm{MeV}$ and 65.0 $\mathrm{MeV}$ at large angles. However the agreement for the proton inelastic analyzing power data with experimental data is not satisfactory.

For ${ }^{28} \mathrm{Si}$, at energies below $20.5 \mathrm{MeV}$, the predictions for proton elastic scattering analyzing power are generally good, but there are obvious deviations at backward angles. And for $65.0 \mathrm{MeV}$ and $80.0 \mathrm{MeV}$, the calculations overestimate the experimental data at angles beyond $60^{\circ}$. The results for proton inelastic scattering analyzing power are smaller than the measurements.

We obtained rather perfect description for proton elastic analyzing power data of ${ }^{60} \mathrm{Ni}$ at overall incident energies and angles. And the predictions for inelastic analyzing data are also good enough. On average, the difference between the calculation and measurement is less than $10 \%$. 
Finally, the predictions of proton elastic analyzing power for ${ }^{90} \mathrm{Zr}$ described the experimental data very well below $79.6 \mathrm{MeV}$. The calculations also described the data well at higher energies, except the deeper extrema. For the inelastic analyzing power, the results are smaller than experimental data.

It can be seen that our potential gives rather good or generally good prediction for proton elastic analyzing power for all nuclei. The proton inelastic analyzing power can be also described in overall agreement for near spherical nuclei, such as ${ }^{60} \mathrm{Ni}$. However for those strong deformed nuclei, such as ${ }^{24} \mathrm{Mg}\left(\beta_{2}=0.5438\right)$ and ${ }^{28} \mathrm{Si}\left(\beta_{2}=-0.4203\right)$, the predictions are a little worse. Considering the fact that the experimental data of analyzing power are not used in the fitting for our potential parameters, while being employed for Koning and Delaroche's spherical potential parameters, it is satisfactory that our global potential can describe simultaneously proton elastic and inelastic scattering analyzing power to such an extent. Our future plan is to incorporate the analyzing power data into OPTMAN code to make more accurate analysis for both angular distributions and analyzing power data simultaneously.

\section{The (p,n) Reaction}

The OPTMAN code had been extended to include the calculation for ( $p, n)$ cross sections with IAS excitation [16], and therefore, making possible to derive Lane consistent potential including a new Coulomb correction. The coupling form factors for chargeexchange calculations are externally calculated as described in Quesada's work. [17].

The results for $\mathrm{Zr}$ isotopes are shown in Fig.5 to demonstrate the predictive power for $(\mathrm{p}, \mathrm{n})$ reaction. The calculations considered the inelastic scattering to the first three levels $0^{+}, 2^{+}, 4^{+}$of the ground-state rotational band and charge-exchange to their IAS. Also all possible pairs of coupling between the target ground state rotational band and IAS had been taken into account. The energy of IAS state was determined by the difference of the experimental Q-value of $(p, n)$ reaction and that of ground state $(p, n)$ reaction. Meanwhile the parameters included in the isovector term of the potentials were slightly adjusted to reproduce the experimental $(\mathrm{p}, \mathrm{n})$ data. However in actual calculations, only those parameters for ${ }^{90} \mathrm{Zr}$ are adjusted, while they were directly used for the (p,n) calculation for ${ }^{92} \mathrm{Zr}$ and ${ }^{94} \mathrm{Zr}$.

It can be seen that the results are in overall good agreement with experimental data for $\mathrm{Zr}$ isotopes, except for a small underestimation of experimental data for ${ }^{94} \mathrm{Zr}$. This means we used right isovector terms as they determine $(\mathrm{A}, \mathrm{Z})$ dependence of the optical potential through $(\mathrm{N}-\mathrm{Z}) / \mathrm{A}$ terms. Therefore we can state that we got a regional potential for $\mathrm{Zr}$ isotopes .

\section{SUMMARY AND CONCLUSION}

The global dispersive coupled-channel optical model potential obtained is used to analyze the analyzing power data and $(\mathrm{p}, \mathrm{n})$ data for some nuclei. The numerical calculations had shown that this potential predicts the proton elastic analyzing power data with general good precision, while the predictions for proton inelastic analyzing power data are slightly worse. The results also showed that present global potential is approximately Lane-consistent, and can predict well the (p,n) cross sections if further adjustment for 
parameters in the isovetor term is done, which encourages further developments to incorporate the analyzing power data as well as $(\mathrm{p}, \mathrm{n})$ data into OPTMAN code to make more accurate analysis for the angular distributions, analyzing power data and $(\mathrm{p}, \mathrm{n})$ data simultaneously.

\section{REFERENCES}

1. L. J. Hao, W. L. Sun and E. Sh. Soukhovitskii, J. Phys. G: Nucl. Part. Phys. 35, 095103(2008)

2. A. J. Koning and J. P. Delaroche, Nucl. Phys. A713, 231(2003)

3. A. M. Lane, Phys. Rev. Lett. 8, 171(1962)

4. E. Sh. Soukhovitskii, R. Capote, J. M. Quesada and S. Chiba, Phys. Rev. C72, 024604(2005)

5. R. H. Bassel, R. M. Drisko and G. R. Satchler, Technical Report ORNL-3240, Oak Ridge National Laboratory Report, 1962

6. E. Sh. Sukhovitskii, S. Chiba, O. Iwamoto and Yu. V. Porodzinskii, Nucl. Phys. A640, 147(1998)

7. C. Mahaux and H. Ngô Nucl. Phys. A431, 486(1984); C. Mahaux, H. Ngô and G. R. Satchler, Nucl. Phys. A449, 354(1986); C. Mahaux and R. Sartor, Nucl. Phys. A468, 193(1987)

8. C. Mahaux and R. Sartor, Nucl. Phys. A484, 205(1988); Nucl. Phys. A493, 157(1989)

9. R. Lipperheide, Z. Phys. 202, 58(1967)

10. R. L. Walter, Proc. Meeting on Nuclon-Nucleus Optical Model up to $200 \mathrm{MeV}$ (France: Bruyèresle-Chatel), November 13-15, p 199, 1996

11. G. E. Brown and M. Rho, Nucl. Phys. A372, 397(1981)

12. J. P. Delaroche, Y. Wang and J. Rapaport, Phys. Rev. C39, 391(1989)

13. E. Sh. Soukhovitskii, S. Chiba, O. Iwamoto, K. Shibata and G. B. Morogovskij, Technical Report JAERI-Data/Code 2005-002, Japan Atomic Energy Research Institute Report, 2005

14. J. Raynal, Notes on ECIS94, CEA Sacley Report No. CEA-N-2772, 1994

15. Available online at http://www-nds.iaea.or.at/exfor/exfor00.htm

16. E. Sh. Soukhovitskii, S. Chiba, R. Capote, J. M. Quesada, S. Kunieda and G. B. Morogovskij, "Supplement to OPTMAN code, Manual Version 10 (2008)", JAEA-Data/Code 2008-025

17. J. M. Quesada, R. Capote, E. Sh. Soukhovitskii, S. Chiba, Phys. Rev. C76, 057602(2007)
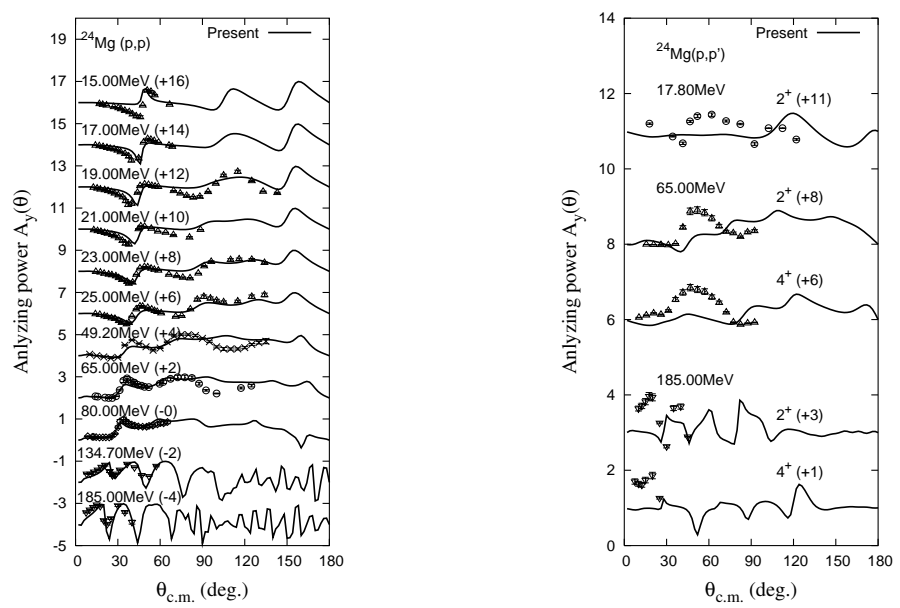

FIGURE 1. Comparison of the predicted and experimental analyzing powers for proton elastic (left) and inelastic scattering (right) from ${ }^{24} \mathrm{Mg}$. The curves and data points are offset by adding 2,4,6 etc to their values. All the experimental data (here and in later figures) are taken from the EXFOR [15] database. 

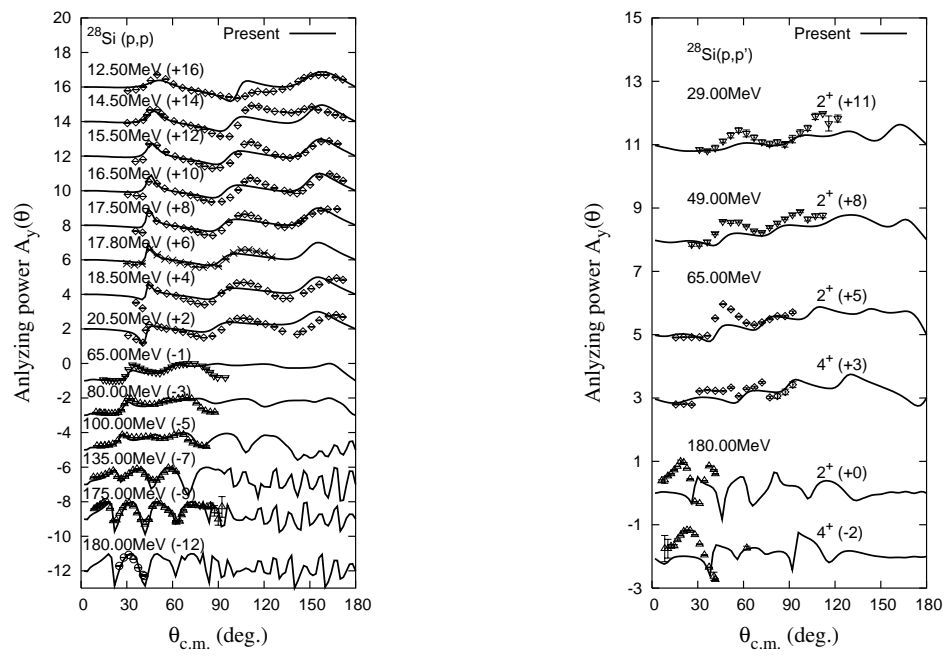

FIGURE 2. Same as Figure 1 but for ${ }^{28} \mathrm{Si}$.
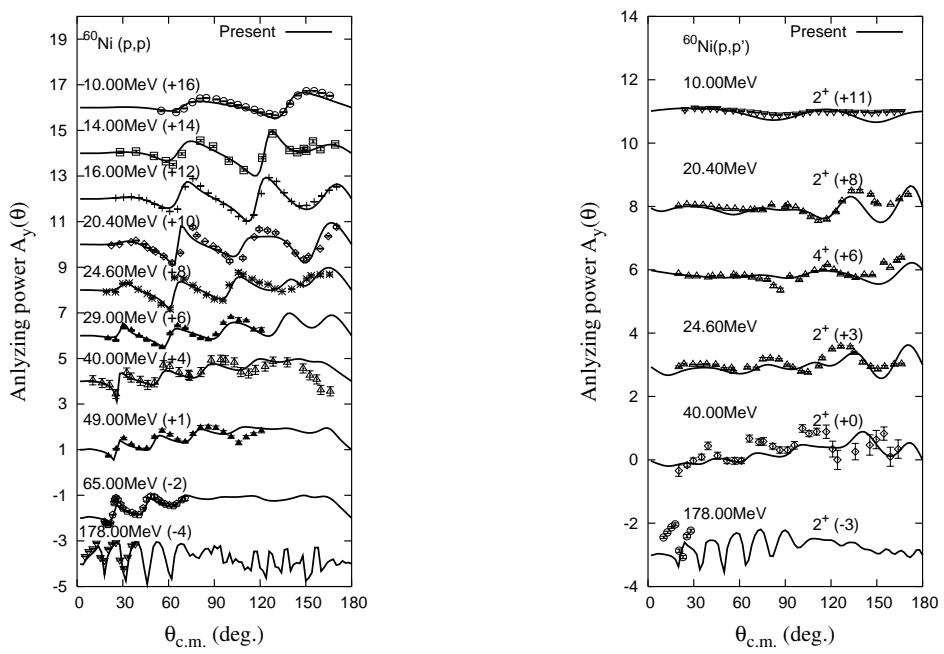

FIGURE 3. Same as Figure 1 but for ${ }^{60} \mathrm{Ni}$. 

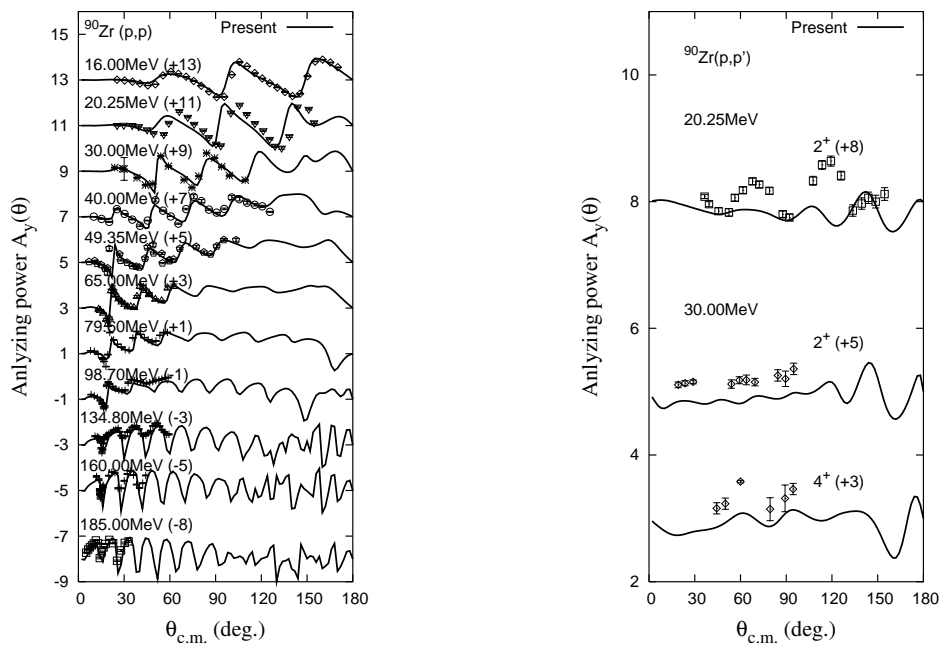

FIGURE 4. Same as Figure 1 but for ${ }^{90} \mathrm{Zr}$.
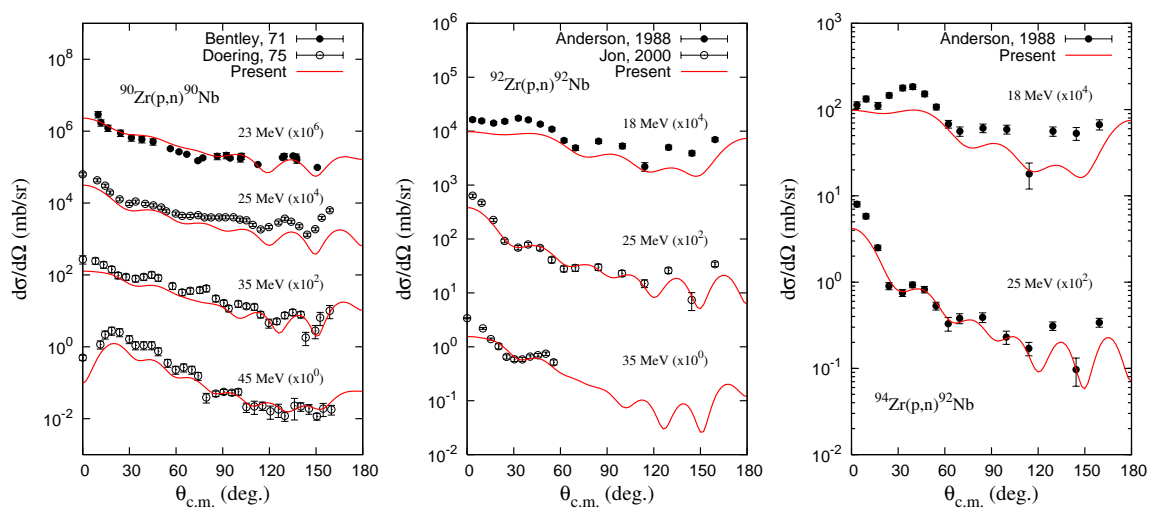

FIGURE 5. Comparison of the predicted and experimental (p, n) cross section for ${ }^{90} \mathrm{Zr}(\mathrm{left})$, ${ }^{90} \mathrm{Zr}$ (middle) and ${ }^{94} \mathrm{Zr}$ (right). 